 \\ TATRA \\ MOUNTaiNS \\ Mathematical Publications}

DOI: $10.2478 / \mathrm{tmmp}-2014-0011$

Tatra Mt. Math. Publ. 58 (2014), 129-136

\title{
NOTES ON MODIFICATIONS OF A wQN-SPACE
}

\author{
JAROSLAV ŠUPINA
}

\begin{abstract}
We continue to investigate the generalizations of the notion of wQN-space introduced by [L. Bukovský-J. Šupina: Modifications of sequence selection principles, Topology Appl. 160 (2013), 2356-2370] and by [J. Šupina: On Ohta-Sakai's properties of a topological space (to appear)]. We present covering characterizations, slightly different formulations, and some new relations among them.
\end{abstract}

\section{Introduction}

All topological spaces are assumed to be infinite and Hausdorff. By a function we mean a real-valued function, and symbol 0 denotes both the number and the function with constant zero value (defined on appropriate topological space). Basic set-theoretical and topological terminology follows mainly [2] and [8]. Preliminary definitions can be found in [1], 3], 12] or in the introduction here.

We continue with investigation of properties introduced in [6] and [16]. Their definitions are generalizations of the definition of wQN-space. Definitions of properties of [16] were motivated by $\mathrm{H}$. O h t a and M. S a k a i 12. To simplify the notation, we need to denote the following preordering on ${ }^{\omega}\left({ }^{X} \mathbb{R}\right)$. Let $\left\langle f_{n} ; n \in \omega\right\rangle$, $\left\langle g_{n} ; n \in \omega\right\rangle$ be two sequences of real-valued functions on $X$. Then we write that $\left\langle f_{n} ; n \in \omega\right\rangle \mathbf{x}^{*}\left\langle g_{n} ; n \in \omega\right\rangle$ if for any $x \in X$ the sequence $\left\{g_{n}(x)\right\}_{n=0}^{\infty}$ eventually dominates sequence $\left\{f_{n}(x)\right\}_{n=0}^{\infty}$, i.e.,

$$
\left\langle f_{n} ; n \in \omega\right\rangle \leq^{*}\left\langle g_{n} ; n \in \omega\right\rangle \equiv(\forall x \in X)\left(\exists n_{0}\right)\left(\forall n \geq n_{0}\right) \quad f_{n}(x) \leq g_{n}(x) .
$$

To make our results easy to formulate, we introduce the following schema which is more general than the schemas of [6] and [16]. Let $X$ be a set, $\mathcal{F}, \mathcal{G}, \mathcal{H} \subseteq X \mathbb{R}$ being families of functions containing the zero constant function, i.e., $0 \in \mathcal{F}, \mathcal{G}, \mathcal{H}$.

(C) 2014 Mathematical Institute, Slovak Academy of Sciences.

2010 Mathematics Subject Classification: Primary 54C50; Secondary 54C30.

Keywords: semicontinuous function, wQN-space.

The work on this research has been partially supported by the grant 1/0002/12 of Slovak Grant Agency VEGA and by P. J. Šafárik University in Košice during author's postdoctoral position. 


\section{JAROSLAV ŠUPINA}

We say that $X$ has a property $\mathbf{w} \mathbf{E D}_{\mathcal{F}, \mathcal{G}}^{\mathcal{H}}$, if for any sequence $\left\langle f_{m} ; m \in \omega\right\rangle$ of functions from $\mathcal{F}$ converging to a function $f \in \mathcal{H}$, there are sequences $\left\langle g_{m} ; m \in \omega\right\rangle$ and $\left\langle h_{m} ; m \in \omega\right\rangle$ of functions from $\mathcal{G}$ converging to $f$, and there is an increasing sequence of natural numbers $\left\{n_{m}\right\}_{m=0}^{\infty}$ such that

for any $x \in X$, the sequence $\left\{g_{m}(x)\right\}_{m=0}^{\infty}$ eventually dominates $\left\{f_{n_{m}}(x)\right\}_{m=0}^{\infty}$, and the sequence $\left\{f_{n_{m}}(x)\right\}_{m=0}^{\infty}$ eventually dominates $\left\{h_{m}(x)\right\}_{m=0}^{\infty}$, i.e.,

$$
\left\langle h_{m} ; m \in \omega\right\rangle \leq^{*}\left\langle f_{n_{m}} ; m \in \omega\right\rangle \leq^{*}\left\langle g_{m} ; m \in \omega\right\rangle .
$$

If $\mathcal{H}=\{0\}$, then we say that $X$ has a property $\mathbf{w E D}_{\mathcal{F}, \mathcal{G}}$. We will use some assumptions about families of functions:
(a) $\{-f ; f \in \mathcal{F}\} \subseteq \mathcal{F}$,
(b) $\{|f| ; f \in \mathcal{F}\} \subseteq \mathcal{F}$,
(c) $\{\min \{f, 1\} ; f \in \mathcal{F}\} \subseteq \mathcal{F}$,
(d) $\{\max \{f, 0\} ; f \in \mathcal{F}\} \subseteq \mathcal{F}$.

For particular families $\mathcal{F}, \mathcal{G}, \mathcal{H} \subseteq{ }^{X} \mathbb{R}$, we obtain some modifications of wQN-space considered earlier. If $\mathcal{F} \subseteq{ }^{X}[0,1]$ and $\mathcal{G}$ satisfies (c), (d), then property $\mathrm{wED}_{\mathcal{F}, \mathcal{G}}$ is equivalent to the property with the same name introduced in [16]. In fact, many pairs $\mathcal{F}, \mathcal{G}$ of families of functions in $\mathrm{wED}_{\mathcal{F}, \mathcal{G}}$ will satisfy the former condition, and therefore, property $\mathrm{wED}_{\mathcal{F}, \mathcal{G}}$ of this paper often corresponds to property $\mathrm{wED}_{\mathcal{F}, \mathcal{G}}$ of [16]. Let Const denote the family of all constant functions on the considered set. For property $\mathrm{wQN}_{\mathcal{F}}$ from [6], we have

$$
\mathrm{wQN}_{\mathcal{F}} \equiv \mathrm{wED}_{\mathcal{F}, \text { Const }} \text {. }
$$

$\mathrm{C}_{p}(X)$ denotes the family of all continuous functions from $X$ to $\mathbb{R}$. L. B u k ovský, I. Recław and M. Repi cký introduced wQN-space in [4] and w $\overline{\mathcal{F}}$ QN-space in [5],

$$
\mathrm{wQN}=\mathrm{wED}_{\mathrm{C}_{p}(X), \text { Const }}, \quad \mathrm{w} \overline{\mathcal{F}} \mathrm{QN}=\mathrm{wED}_{\mathcal{F}, \text { Const }}^{X_{\mathbb{R}}} .
$$

L. Bukovský [1] introduced $\mathrm{wQN}^{*}$-space and $\mathrm{wQN}_{*}$-space 1$] \mathcal{U}$ and $\mathcal{L}$ are families of all upper and lower semicontinuous functions on $X$, respectively. For a family $\mathcal{F} \subseteq \subseteq^{X}$ we denote $\widetilde{\mathcal{F}}=\mathcal{F} \cap X[0,1]$. Then,

$$
\mathrm{wQN}^{*} \equiv \mathrm{wED}_{\widetilde{\mathcal{U}}, \text { Const }}, \quad \mathrm{wQN}_{*} \equiv \mathrm{wED}_{\widetilde{\mathcal{L}}, \text { Const }} .
$$

We begin with simple properties of $\mathrm{wED}_{\mathcal{F}, \mathcal{G}}^{\mathcal{H}}$. If $X$ is any set and $\mathcal{G} \subseteq \mathcal{F} \subseteq{ }^{X} \mathbb{R}$, $\mathcal{H} \subseteq{ }^{X} \mathbb{R}$, then property ${ }^{{ }_{2}} \mathrm{ED}_{\mathcal{F}, \mathcal{G}}^{\mathcal{H}}$ is trivially satisfied in $X$. If $\mathcal{F}_{1} \subseteq \mathcal{F}_{2}, \mathcal{G}_{1} \subseteq \mathcal{G}_{2}$ and $\mathcal{H}_{1} \subseteq \mathcal{H}_{2}$, then

$$
\mathrm{wED}_{\mathcal{F}_{2}, \mathcal{G}_{1}}^{\mathcal{H}_{2}} \rightarrow \mathrm{wED}_{\mathcal{F}_{1}, \mathcal{G}_{2}}^{\mathcal{H}_{1}}
$$

By $\mathcal{B}$ we denote the family of all Borel functions on $X$. If $X$ is a perfectly normal space, $\mathcal{F}, \mathcal{H} \subseteq \mathcal{B}$ and Const $\subseteq \mathcal{G}$ then, in accordance with Tsaban-Zdomskyy Theorem [17], we have

$$
\mathrm{QN} \rightarrow \mathrm{wED}_{\mathcal{B}, \text { Const }}^{\mathcal{B}} \rightarrow \mathrm{wED}_{\mathcal{F}, \mathcal{G}}^{\mathcal{H}}
$$

\footnotetext{
${ }^{1}$ For more information about values of functions in their definitions see [15].
} 


\section{NOTES ON MODIFICATIONS}

If a family $\mathcal{F}$ satisfies (b) and $\mathcal{G}$ satisfies (a), (d), then

$$
\mathrm{wED}_{\mathcal{F}, \mathcal{G}} \equiv \mathrm{wED}_{\mathcal{F} \cap X}[0, \infty), \mathcal{G} \cap x[0, \infty) .
$$

If a family $\mathcal{F}$ satisfies (b), (c) and $\mathcal{G}$ satisfies (a), (c), (d), then

$$
\mathrm{wED}_{\mathcal{F}, \mathcal{G}} \equiv \mathrm{wED}_{\widetilde{\mathcal{F}}, \widetilde{\mathcal{G}}} \text {. }
$$

Finally, if $\mathcal{F} \subseteq{ }^{X}[0,1]$ and $\mathcal{G}$ satisfies (c), (d), then

$$
\mathrm{wED}_{\mathcal{F}, \mathcal{G}} \equiv \mathrm{wED}_{\mathcal{F}, \widetilde{\mathcal{G}}} \text {. }
$$

We will use these relations without any comment.

\section{Coverings}

There are already known covering characterizations of some properties $\mathrm{wED}_{\mathcal{F}, \mathcal{G}}$. Such a characterization for wQN-space was found in [3]. By [1], [3], 13], property $\alpha_{1}(\Gamma, \Gamma)$ in the sense of $\mathrm{L} \mathrm{j} . \mathrm{D}$. R. K o čin a c [10] is a characterization of $\mathrm{wED}_{\widetilde{\mathcal{L}}, \text { Const }}$. In [16], we found covering characterizations of $\mathrm{wED}_{\widetilde{\mathcal{L}}, \mathrm{C}_{p}(X)}$ and ${ }_{\mathrm{w}} \mathrm{ED}_{\tilde{\mathcal{U}}, \mathrm{C}_{p}(X)} \cdot$

A family $\mathcal{A} \subseteq \mathcal{P}(X)$ is a cover of a topological space $X$ if $X=\bigcup \mathcal{A}$ and $X \notin \mathcal{A} 2$ An infinite cover $\mathcal{A}$ is a $\gamma$-cover if every $x \in X$ lies in all but finitely many members of $\mathcal{A}$. $\Gamma$ denotes the family of all open $\gamma$-covers of $X$. L. B u k o vský [1] showed that any $\mathrm{S}_{1}(\Gamma, \Gamma)$-space has $\mathrm{wED}_{\tilde{\mathcal{U}} \text {, Const }}$, and M. Sakai [14] proved that if a topological space $X$ has $\mathrm{wED}_{\tilde{\mathcal{U}} \text {,Const }}$, then $X$ is an $\mathrm{S}_{1}(\Gamma, \Gamma)$-space. We prove generalizations of these results using ideas of their proofs.

Let $X$ be a set and $\mathcal{A} \subseteq \mathcal{P}(X)$. $\mathcal{A}^{c}$ denotes the family $\{X \backslash A ; A \in \mathcal{A}\}$. By $\Gamma_{\mathcal{A}}$ we mean the family of all $\gamma$-covers of $X$ by sets from $\mathcal{A}$. A function $f$ on $X$ is called lower, upper $\mathcal{A}$-measurable if $f^{-1}((r, \infty)) \in \mathcal{A}, f^{-1}((-\infty, r)) \in \mathcal{A}$ for any $r \in \mathbb{R}$, respectively. The family of all lower, upper $\mathcal{A}$-measurable functions on $X$ with values in $[0,1]$ is denoted by $\mathcal{L}(\mathcal{A}), \mathcal{U}(\mathcal{A})$, respectively.

Theorem 2.1. Let $X$ be a topological space, $\mathcal{A} \subseteq \mathcal{P}(X)$ being closed under finite unions and intersections 3 Then the following are equivalent.

(1) $X$ is an $\mathrm{S}_{1}\left(\Gamma_{\mathcal{A}}, \Gamma_{\mathcal{A}}\right)$-space.

(2) $X$ has $\mathrm{wED}_{\mathcal{U}(\mathcal{A}) \text {, Const }}$.

(3) $X$ has $\mathrm{wED}_{\mathcal{L}\left(\mathcal{A}^{c}\right) \text {, Const }}$.

\footnotetext{
${ }^{2}$ Similarly to [3], the empty set $\emptyset$ can be an element of a cover. If we consider the enumeration of a cover, then we always assume that the set is repeated only finitely many times in the enumeration, i.e., the enumeration is adequate in the sense of [2].

${ }^{3}$ Thus $\emptyset, X \in \mathcal{A}$.
} 
P r o of. $(1) \rightarrow(2)$ Let $\left\langle f_{m} ; m \in \omega\right\rangle$ be a sequence of upper $\mathcal{A}$-measurable functions on $X$ with values in $[0,1]$ such that $f_{m} \rightarrow 0$. We define the sets $A_{n, m}, n$, $m \in \omega$ by

$$
A_{n, m}=\left\{x \in X ; f_{m}(x)<2^{-n}\right\} .
$$

If there are increasing sequences $\left\{n_{k}\right\}_{k=0}^{\infty},\left\{m_{k}\right\}_{k=0}^{\infty}$ such that $A_{n_{k}, m_{k}}=X$ for all $k \in \omega$, then $\left\langle f_{m_{k}} ; k \in \omega\right\rangle$ converges uniformly. Thus, we may assume that $\left\langle\left\{A_{n, m} ; m \in \omega\right\} ; n \in \omega\right\rangle$ is a sequence of $\gamma$-covers by sets from $\mathcal{A}$. By $\mathrm{S}_{1}\left(\Gamma_{\mathcal{A}}, \Gamma_{\mathcal{A}}\right)$ there is an increasing sequence $\left\{m_{n}\right\}_{n=0}^{\infty}$ such that $\left\{A_{n, m_{n}} ; n \in \omega\right\}$ is a $\gamma$-cover enumerated bijectively. Then, $\left\langle f_{m_{n}} ; n \in \omega\right\rangle$ converges quasi-normally to zero with the control $\left\{2^{-n}\right\}_{n=0}^{\infty}$.

$(2) \rightarrow(1)$ Let $\left\langle\left\{A_{n, m} ; m \in \omega\right\} ; n \in \omega\right\rangle$ be a sequence of $\gamma$-covers by sets from $\mathcal{A}$. Since $\mathcal{A}$ is closed under finite intersections, we may assume that $A_{n+1, m} \subseteq A_{n, m}$ for any $n, m \in \omega$. We define the upper $\mathcal{A}$-measurable functions $f_{m}, m \in \omega$ by

$$
f_{m}(x)= \begin{cases}1, & x \in X \backslash A_{0, m} \\ \frac{1}{2^{n+1}}, & x \in A_{n, m} \backslash A_{n+1, m}, n \in \omega, \\ 0, & \text { otherwise. }\end{cases}
$$

Sequence $\left\langle f_{m} ; m \in \omega\right\rangle$ converges to zero. We have $f_{m}(x)<\frac{1}{2^{n}}$ if and only if $x \in A_{n, m}$. By $\mathrm{wED}_{\mathcal{U}(\mathcal{A}) \text {, Const }}$ there is an increasing sequence $\left\{m_{n}\right\}_{n=0}^{\infty}$ such that $\left\langle f_{m_{n}} ; n \in \omega\right\rangle$ converges quasi-normally to zero with the control $\left\{2^{-n}\right\}_{n=0}^{\infty}$. Thus, $\left\{A_{n, m_{n}} ; n \in \omega\right\}$ is a $\gamma$-cover (by respective reselection we may assume that the enumeration is bijective).

The equivalence of (1) and (3) can be proved similarly.

Let us denote by $\mathbf{F}$ the family of all closed subsets of $X$. Then, we have Corollary 2.2. Note that according to B. Ts a ban and L. Zdomsky y 17] and L. Bukovský [1] the result is known for perfectly normal space.

Corollary 2.2. A topological space $X$ has $\mathrm{wED}_{\widetilde{\mathcal{L}} \text {,Const }}$ if and only if $X$ is an $\mathrm{S}_{1}\left(\Gamma_{\mathbf{F}}, \Gamma_{\mathbf{F}}\right)$-space.

Note that the paper [6] contains characterizations of properties of Theorem 2.1 by so-called sequence selection properties.

\section{Various families of functions}

The paper [6] contains relations among properties $\mathrm{wED}_{\mathcal{F}, \text { Const }}$ for various interesting families $\mathcal{F}$, e.g., if $\widetilde{\mathcal{L}} \subseteq \mathcal{F} \subseteq \mathcal{B}$, then we have

$$
\mathrm{QN} \equiv \mathrm{wED}_{\mathcal{F}, \text { Const }}, \quad \mathrm{S}_{1}(\Gamma, \Gamma) \equiv \mathrm{wED}_{\widetilde{\mathcal{U}}, \text { Const }} .
$$

We accomplish the similar investigations of properties $\mathrm{wED}_{\widetilde{\mathcal{F}}, \mathrm{C}_{p}(X)}$ and $\mathrm{wED}_{\widetilde{\mathcal{F}}, \mathcal{U}}$. For interesting families, these properties can be divided into two groups of equivalent properties. By $\mathcal{B}_{1}$ we denote the family of all pointwise limits of continuous 


\section{NOTES ON MODIFICATIONS}

functions on $X$. If $X$ is a perfectly normal space, then $\mathcal{B}_{1}$ is the family of all $\mathrm{F}_{\sigma}$-measurable functions on $X$.

TheOREM 3.1. Let $X$ be a perfectly normal space.

(1) $X$ has $\mathrm{wED}_{\widetilde{\mathcal{L}}, \mathrm{C}_{p}(X)}$ if and only if $X$ has $\mathrm{wED}_{\widetilde{\mathcal{L}}, \mathcal{U}}$.

(2) If $\widetilde{\mathcal{B}}_{1} \subseteq \mathcal{F} \subseteq \widetilde{\mathcal{B}}$, then

Proof.

$$
\mathrm{wED}_{\mathcal{B}, \mathrm{C}_{p}(X)} \equiv \mathrm{wED}_{\mathcal{F}, \mathrm{C}_{p}(X)} \equiv \mathrm{wED}_{\mathcal{F}, \mathcal{U}} .
$$

(1) Let us assume that $X$ has $\operatorname{wED}_{\widetilde{\mathcal{L}}, \mathcal{U}}$. By [16, Corollary 5.2] we have that $X$ has $\mathrm{wED}_{\widetilde{\mathcal{U}}, \mathrm{C}_{p}(X)}$. One can easily see that if $X$ has $\mathrm{wED}_{\widetilde{\mathcal{L}}, \mathcal{U}}$ and $\mathrm{wED}_{\tilde{\mathcal{U}}, \mathrm{C}_{p}(X)}$, then $X$ has $\mathrm{wED}_{\widetilde{\mathcal{L}}, \mathrm{C}_{p}(X)}[16$, Lemma 2.2].

(2) Similarly to (1), one can show that if $X$ has $\operatorname{wED}_{\mathcal{F}, \mathcal{U}}$, then $X$ has

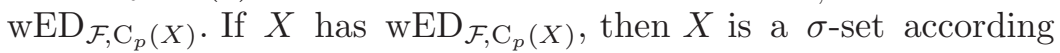
to [16, Corollary 5.2]. Therefore, any Borel function is $\mathrm{F}_{\sigma}$-measurable and belongs to family $\mathcal{F}$. Hence, $X$ has ${ }^{w E D} \mathrm{E}_{\widetilde{\mathcal{B}}, \mathrm{C}_{p}(X)}$.

However, following in [16, Theorem 1.2], we have

Proposition 3.2. Let $X$ be a perfectly normal space with Hurewicz property $\widetilde{\mathcal{L}} \subseteq \mathcal{F} \subseteq \widetilde{\mathcal{B}}$. Then

$$
\mathrm{QN} \equiv \mathrm{wED}_{\mathcal{F}, \mathrm{C}_{p}(X)} \equiv \mathrm{wED}_{\mathcal{F}, \mathcal{U}} .
$$

In [16], we showed that, for any perfectly normal space, the property $\mathrm{wED}_{\widetilde{\mathcal{L}}, \mathrm{C}_{p}(X)}$ is hereditary. The same is true for $\mathrm{wED}_{\mathcal{B}, \mathrm{C}_{p}(X)}$.

Lemma 3.3. Let $X$ be a topological space, and let $\mathcal{G} \in\left\{\mathrm{C}_{p}(X), \mathcal{U}, \mathcal{L}, \mathcal{B}_{1}\right\}$. Then, any Borel subset of $X$ with property $\mathrm{wED}_{\widetilde{\mathcal{B}}, \mathcal{G}}$ has $\mathrm{wED}_{\widetilde{\mathcal{B}}, \mathcal{G}}$ as well.

Proof. Let $B \subseteq X$. For a sequence $\left\langle f_{n} ; n \in \omega\right\rangle$ of Borel functions on $B$, one can define a sequence $\left\langle h_{n} ; n \in \omega\right\rangle$ of Borel functions on $X$ by $h_{n}(x)=f_{n}(x)$ for $x \in B$ and $h_{n}(x)=0$ for $x \in X \backslash B$.

If $X$ is perfectly normal space, then by Kuratowski Extension Theorem for Borel measurable functions (see, e.g., [11, §31, VI, Théorème] or [7, Theorem 2.4]) we obtain

Proposition 3.4. For any perfectly normal space $X$, the property $\mathrm{wED}_{\mathcal{B}, \mathrm{C}_{p}(X)}$ is hereditary.

\footnotetext{
${ }^{4}$ We say that a topological space $X$ possesses Hurewicz property if for any sequence $\left\langle\mathcal{U}_{n} ; n \in \omega\right\rangle$ of countable open covers not containing a finite subcover, there exist finite sets $\mathcal{V}_{n} \subseteq \mathcal{U}_{n}, n \in \omega$ such that $\left\{\bigcup \mathcal{V}_{n} ; n \in \omega\right\}$ is a $\gamma$-cover. Note that this definition corresponds to property $\mathrm{E}_{\omega}^{* *}$ rather than to original property $\mathrm{E}^{* *}$ by W. Hurewicz [9], see, e.g., [3].
} 


\section{Different formulations}

In [15], we showed that the range of functions in definitions of properties ${ } \mathrm{ED}_{\widetilde{\mathcal{U}}, \text { Const }}$ and $\mathrm{wED}_{\widetilde{\mathcal{L}} \text {, Const }}$ is essential, e.g., if $X$ is a normal space, then

$$
\mathrm{wED}_{\widetilde{\mathcal{L}}, \text { Const }} \equiv \mathrm{wED}_{\mathcal{L}, \text { Const }} \equiv \mathrm{wED}_{\mathcal{U}, \text { Const }} .
$$

In this section, we present similar, but not the same, results on main objects of investigation in [16], i.e., on properties $\mathrm{wED}_{\widetilde{\mathcal{U}}, \mathrm{C}_{p}(X)}$ and $\mathrm{wED}_{\widetilde{\mathcal{L}}, \mathrm{C}_{p}(X)}$.

For a perfectly normal space $X$, we show that the limit function in the definition of property $\mathrm{wED}_{\widetilde{\mathcal{U}}, \mathrm{C}_{p}(X)}$ can be any $\mathrm{F}_{\sigma}$-measurable function, and the range of functions can be $\mathbb{R}$.

Proposition 4.1. Let $X$ be a perfectly normal space. The following are equivalent.

(1) X possesses $\mathrm{wED}_{\tilde{\mathcal{U}}, \mathrm{C}_{p}(X)}$.

(2) For any sequence $\left\langle f_{m} ; m \in \omega\right\rangle$ of upper semicontinuous functions on $X$ with values in $\mathbb{R}$ converging to $\mathrm{F}_{\sigma}$-measurable function $f$, there exists a sequence $\left\langle g_{m} ; m \in \omega\right\rangle$ of continuous functions converging to $f$, and there is an increasing sequence of natural numbers $\left\{n_{m}\right\}_{m=0}^{\infty}$ such that

$$
\left\langle f_{n_{m}} ; m \in \omega\right\rangle \leq^{*}\left\langle g_{m} ; m \in \omega\right\rangle .
$$

Proof. Since there is an increasing homeomorphism between $(0,1)$ and $\mathbb{R}$, we will restrict our proof to functions with $(0,1)$ range. Thus, let $\left\langle f_{m} ; m \in \omega\right\rangle$ be a sequence of upper semicontinuous functions on $X$ with values in $(0,1)$ converging to an $\mathrm{F}_{\sigma}$-measurable function $f$, and let $\left\langle h_{n} ; n \in \omega\right\rangle$ be continuous functions such that $h_{n} \rightarrow f$. Then, $\max \left\{f_{n}-h_{n} ; 0\right\} \rightarrow 0$. In accordance with wED $\tilde{\mathcal{U}}, \mathrm{C}_{p}(X)$, there exist a sequence $\left\langle g_{m}^{\prime} ; m \in \omega\right\rangle$ of continuous functions converging to zero and an increasing sequence of natural numbers $\left\{n_{m}\right\}_{m=0}^{\infty}$ such that for any $x \in X$ there is $m_{0} \in \omega$ with $\max \left\{f_{n_{m}}(x)-h_{n_{m}}(x) ; 0\right\} \leq g_{m}^{\prime}(x)$ for any $m \geq m_{0}$. Then, $f_{n_{m}}(x) \leq g_{m}^{\prime}(x)+h_{n_{m}}(x)$ for any $m \geq m_{0}$.

Finally, we define a sequence $\left\langle g_{m} ; m \in \omega\right\rangle$ by

$$
g_{m}=\min \left\{1-2^{-m-1} ; \max \left\{2^{-m-1} ; g_{m}^{\prime}+h_{n_{m}}\right\}\right\}
$$

and we obtain functions with ranges in $(0,1)$ such that

$$
\left\langle f_{n_{m}} ; m \in \omega\right\rangle \leq^{*}\left\langle g_{m} ; m \in \omega\right\rangle \text {. }
$$

Although the condition (2) of Proposition 4.1 resembles property $\mathrm{wED}_{\mathcal{U}, \mathrm{C}_{p}(X)}^{\mathcal{B}_{1}}$, it cannot be replaced with this property, as Theorem 4.2 shows.

Let property $\operatorname{ED}_{\mathcal{F}, \mathcal{G}}^{\mathcal{H}}$ be defined as property $\mathrm{wED}_{\mathcal{F}, \mathcal{G}}^{\mathcal{H}}$, except for the condition asking $\left\langle h_{m} ; m \in \omega\right\rangle \leq^{*}\left\langle f_{m} ; m \in \omega\right\rangle \leq^{*}\left\langle g_{m} ; m \in \omega\right\rangle$. In [16], we showed that a topological space $X$ has $\mathrm{wED}_{\widetilde{\mathcal{L}}, \mathrm{C}_{p}(X)}$ if and only if $X$ has $\mathrm{ED}_{\widetilde{\mathcal{L}}, \mathrm{C}_{p}(X)}$. 


\section{NOTES ON MODIFICATIONS}

TheOREM 4.2. Let $X$ be a perfectly normal space. Then

$$
\mathrm{wED}_{\widetilde{\mathcal{L}}, \mathrm{C}_{p}(X)} \equiv \mathrm{wED}_{\mathcal{L}, \mathrm{C}_{p}(X)}^{\mathcal{B}} \equiv \mathrm{wED}_{\mathcal{U}, \mathrm{C}_{p}(X)}^{\mathcal{B}} .
$$

Pr o o f. If $X$ has $\mathrm{wED}_{\mathcal{L}, \mathrm{C}_{p}(X)}^{\mathcal{B}}$, then $X$ has $\mathrm{wED}_{\widetilde{\mathcal{L}}, \mathrm{C}_{p}(X)}$ as well. Since there is an increasing homeomorphism between $(0,1)$ and $\mathbb{R}$, to prove the reversed implication, we will restrict to the functions with $(0,1)$ range. Let $X$ possess ${ }^{w E D} D_{\widetilde{\mathcal{L}}, \mathrm{C}_{p}(X)}$, and let $\left\langle f_{m} ; m \in \omega\right\rangle$ be a sequence of upper semicontinuous functions on $X$ with values in $(0,1)$ converging to $f \in \mathcal{B}$. By [16. Corollary 5.2], the function $f$ is $\boldsymbol{\Delta}_{2}^{0}$-measurable, thus $f \in \mathcal{B}_{1}$. Moreover, $X$ has USC by the same corollary and [12, Corollary 2.4]. Thus, by [16, Theorem 6.1], there is a sequence $\left\langle\varphi_{m} ; m \in \omega\right\rangle$ of continuous functions converging to $f$ such that $f_{m} \leq \varphi_{m}$. Taking the lower semicontinuous functions $\varphi_{m}-f_{m}$, we have $\varphi_{m}-f_{m} \rightarrow 0$. Due to ${ }_{\text {wED }}{\mathrm{\mathcal {L }}, \mathrm{C}_{p}(X)}$, there is a sequence $\left\langle\psi_{m} ; m \in \omega\right\rangle$ of continuous functions converging to zero such that $\left\langle\varphi_{m}-f_{m} ; m \in \omega\right\rangle \leq^{*}\left\langle\psi_{m} ; m \in \omega\right\rangle$. The sequences $\left\langle h_{m} ; m \in \omega\right\rangle$ and $\left\langle g_{m} ; m \in \omega\right\rangle$ will be defined by

$$
h_{m}=\max \left\{2^{-m-1} ; \varphi_{m}-\psi_{m}\right\}, g_{m}=\min \left\{1-2^{-m-1} ; \varphi_{m}\right\} .
$$

To prove the equivalence $\mathrm{wED}_{\mathcal{L}, \mathrm{C}_{p}(X)}^{\mathcal{B}} \equiv \mathrm{wED}_{\mathcal{U}, \mathrm{C}_{p}(X)}^{\mathcal{B}}$ for a sequence $\left\langle f_{m} ; m \in \omega\right\rangle$ of upper/lower semicontinuous functions converging to a Borel function $f$, we can consider the lower/upper semicontinuous functions $-f_{m}, m \in \omega$ and the Borel function $-f$.

Let us remark that

$$
\mathrm{wED}_{\widetilde{\mathcal{L}}, \mathrm{C}_{p}(X)}^{\mathcal{\mathcal { B }}} \equiv \mathrm{wED}_{\widetilde{\mathcal{U}}, \mathrm{C}_{p}(X)}^{\mathcal{\mathcal { B }}} .
$$

To prove this, one can use functions $1-f_{m}, m \in \omega$ and $1-f$ instead of $-f_{m}, m \in \omega$ and $-f$ in the second part of the proof of Theorem 4.2 .

In fact, notice that slightly more is proved in Theorem 4.2, i.e.,

$$
{ } \mathrm{ED}_{\widetilde{\mathcal{L}}, \mathrm{C}_{p}(X)} \equiv \operatorname{ED}_{\mathcal{L}, \mathrm{C}_{p}(X)}^{\mathcal{B}} \equiv \operatorname{ED}_{\mathcal{U}, \mathrm{C}_{p}(X)}^{\mathcal{B}}
$$

Consequently, for any $\{0\} \subseteq \mathcal{F} \subseteq \mathcal{B}$, we obtain

$$
\mathrm{wED}_{\widetilde{\mathcal{L}}, \mathrm{C}_{p}(X)} \equiv \mathrm{wED}_{\mathcal{L}, \mathrm{C}_{p}(X)}^{\mathcal{F}} \equiv \mathrm{wED}_{\mathcal{U}, \mathrm{C}_{p}(X)}^{\mathcal{F}} \equiv \mathrm{ED}_{\mathcal{L}, \mathrm{C}_{p}(X)}^{\mathcal{F}} \equiv \mathrm{ED}_{\mathcal{U}, \mathrm{C}_{p}(X)}^{\mathcal{F}}
$$

Finally, note that, for perfectly normal space $X$ and $\widetilde{\mathcal{B}}_{1} \subseteq \mathcal{F} \subseteq \mathcal{B}$, we have

$$
\mathrm{wED}_{\mathcal{B}, \mathrm{C}_{p}(X)} \equiv \mathrm{wED}_{\mathcal{F}, \mathrm{C}_{p}(X)}^{\mathcal{B}} .
$$

Acknowledgements. I would like to thank Professor L ev Buk ovský and the referee for valuable comments. 


\section{JAROSLAV ŠUPINA}

\section{REFERENCES}

[1] BUKOVSKÝ, L.: On $\mathrm{wQN}_{*}$ and $\mathrm{wQN}^{*}$ spaces, Topology Appl. 156 (2008), 24-27.

[2] BUKOVSKÝ, L.: The Structure of the Real Line, in: Monogr. Mat., Vol. 71, Birkhäuser, Basel, 2011.

[3] BUKOVSKÝ, L.-HALEŠ, J.: QN-spaces, wQN-spaces and covering properties, Topology Appl. 154 (2007), 848-858.

[4] BUKOVSKÝ, L.-RECŁAW, I.-REPICKÝ, M.: Spaces not distinguishing pointwise and quasinormal convergence of real functions, Topology Appl. 41 (1991), 25-40.

[5] BUKOVSKÝ, L.-RECŁAW, I.-REPICKÝ, M.: Spaces not distinguishing convergences of real-valued functions, Topology Appl. 112 (2001), 13-40.

[6] BUKOVSKÝ, L.-ŠUPINA, J.: Modifications of sequence selection principles, Topology Appl. 160 (2013), 2356-2370.

[7] CSÁSZÁR, Á.: Extensions of discrete and equal Baire functions, Acta Math. Hungar. 56 (1990), 93-99.

[8] ENGELKING, R.: General Topology. Heldermann, Berlin, 1989.

[9] HUREWICZ, W.: Über Folgen stetiger Funktionen, Fund. Math. 9 (1927), 193-204.

[10] KOČINAC, LJ. D. R.: Selection principles related to $\alpha_{i}$-properties, Taiwanese J. Math. 12 (2008), 561-571.

[11] KURATOWSKI, K.: Topologie I, in: Monogr. Mat., Vol. 20, Warszawa, 1948.

[12] OHTA, H.-SAKAI, M.: Sequences of semicontinuous functions accompanying continuous functions, Topology Appl. 156 (2009), 2683-2906.

[13] SAKAI, M.: The sequence selection properties of $\mathrm{C}_{p}(X)$, Topology Appl. 154 (2007), 552-560.

[14] SAKAI, M.: Selection principles and upper semicontinuous functions, Colloq. Math. 117 (2009), 251-256.

[15] ŠUPINA, J.: wQN spaces and related notions, Tatra Mt. Math. Publ. 46 (2010), 71-77.

[16] ŠUPINA, J.: On Ohta-Sakai's properties of a topological space (to appear).

[17] TSABAN, B.-ZDOMSKYY, L.: Hereditary Hurewicz spaces and Arhangel'skiน sheaf amalgamations, J. Eur. Math. Soc. (JEMS) 14 (2012), 353-372.

Received December 2, 2013

Institute of Mathematics

P. J. Šafárik University in Košice

Jesenná 5

SK-040-01 Košice

SLOVAKIA

E-mail: jaroslav.supina@upjs.sk 